Int. J. Contemp. Math. Sci., Vol. 2, 2007, no. 13, 615 - 625

\title{
Evaluation Codes
}

Associated to Some Matrices

\author{
M. González Sarabia ${ }^{1}$ \\ Unidad Profesional Interdisciplinaria \\ en Ingeniería y Tecnologías Avanzadas \\ Instituto Politécnico Nacional \\ 07340 México, D.F., MEXICO \\ e-mail: msarabia@itesm.mx \\ C. Rentería M. ${ }^{2}$ \\ Escuela Superior de Física y Matemáticas \\ Instituto Politécnico Nacional \\ 07300 México, D.F., MEXICO \\ e-mail: renteri@esfm.ipn.mx
}

\begin{abstract}
In this paper we define the evaluation codes associated to some specific matrices which are related to toric varieties. We compute the main parameters of these codes: the length, the dimension and the minimum distance. It is very important to say that these codes are MDS codes. Finally, we give two specific examples where these characteristics are described
\end{abstract}

Mathematics Subject Classification: 94B27, 94B60

Keywords: Evaluation code, $a$-invariant, toric variety, MDS code

\section{Introduction}

We introduce a general method to construct evaluation linear codes associated to some matrices, this construction generalize most of the cases studied earlier, for the matrix studied here we prove that the associated code is an MDS code.

\footnotetext{
${ }^{1}$ Partially supported by COFFA-IPN and SNI-SEP, México.

${ }^{2}$ Partially supported by COFFA-IPN and SNI-SEP, México.
} 


\section{Evaluation Codes}

Let $K$ be a finite field with $q$ elements, let $\mathbb{P}_{K}^{l}$ be the $l$ - projective space over $K$ and $X=\left\{P_{1}, \ldots, P_{s}\right\}$ be a subset of $\mathbb{P}_{K}^{l}$. We always use the standard representation for the points in $\mathbb{P}_{K}^{l}$, i.e., $P=\left(0,0, \ldots, 0,1, a_{i}, \ldots, a_{l}\right)$. Let $\mathcal{L}$ be a finite dimensional $K$ - linear space of functions which are defined on the set $X$ and take values on $K$. Thus the evaluation map

$$
\begin{gathered}
e v: \mathcal{L} \rightarrow K^{s}, \\
e v(f)=\left(f\left(P_{1}\right), \ldots, f\left(P_{s}\right)\right)
\end{gathered}
$$

defines a $K$-linear code: $C_{X}=e v(\mathcal{L})$.

Let $S=K\left[X_{0}, \ldots, X_{l}\right]=\bigoplus_{d>0} S_{d}$ be the polynomial ring over the finite field $K$ with the natural graduation. If $\mathcal{L}=S_{d}$ is the $d$-graded homogeneous component of the polynomial ring $S$, the corresponding linear code $C_{X}(d):=e v\left(S_{d}\right)$ will be called the evaluation linear code over the set $X$, which is isomorphic to $S_{d} / I_{X}(d)$, where $I_{X}=\bigoplus_{d \geq 0} I_{X}(d)$ is the graded vanishing ideal of $X$. The dimension of these codes is given by the Hilbert function of $S / I_{X}$, i.e., $\operatorname{dim}_{K} C_{X}(d)=H_{X}(d)$.

This kind of codes has been studied in many particular cases (cf. [1], [2], [4], [5], [6], [7],[9], [10], [11],[12], [13]) and their main parameters have been computed. Especific examples have been given with the help of Macaulay 2 (cf. [8]). In fact, a generating matrix of these codes can be obtained by finding a Gröbner basis for the ideal $I_{X}$, and then the cosets module $I_{X}(d)$ of monomials of degree $d$ not belonging to the leading terms ideal $L T\left(I_{X}\right)$ of $I_{X}$, forms a $K$-basis for $S_{d} / I_{X}(d)$. If $B \subseteq S_{d}$ is this set of monomials then $(e v(h))_{h \in B}$ is a generating matrix for $C_{X}(d)$.

\section{$2.1 \quad a-$ Invariant}

Let $I_{X}=\bigoplus_{d=\gamma}^{\infty} I_{X}(d)$ with $I_{X}(\gamma) \neq 0$, so that $\gamma$ is the lowest degree of a nontrivial homogeneous component of the ideal $I_{X}$. There is an integer $a_{X}$ called the $a$-invariant of $S / I_{X}$ (or the $a$-invariant of the ideal $I_{X}$ or even the $a$-invariant of $X$ ) such that

(1) $H_{X}(d)=\operatorname{dim}_{K} S_{d}=\left(\begin{array}{c}d+l \\ l\end{array}\right)$ if and only if $d<\gamma$;

(2) $H_{X}(d)<H_{X}(d+1)<s$ for $0 \leq d<a_{X}$;

(3) $H_{X}(d)=s$ for $d>a_{X}$.

The number $a_{X}+1$ is called the regularity index of $S / I_{X}$ or $I_{X}$. Moreover, the vanishing ideal $I_{X}$ is given by

$$
I_{X}=\left\langle I_{X}(\gamma), I_{X}(\gamma+1), \ldots, I_{X}\left(a_{X}+2\right)\right\rangle
$$




\subsection{Toric Varieties}

Let $A=\left(a_{i j}\right)$ be a fixed $m \times(n+1)$ matrix with non negative integer entries $a_{i j}$ and with non-zero columns. Let $K\left[X_{0}, \ldots, X_{n}\right]$ and $K\left[t_{1}, \ldots, t_{m}\right]$ the two polynomial rings over $K$, and $\varphi$ the graded homomorphism of $K$-algebras

$$
\varphi: K\left[X_{0}, \ldots, X_{n}\right] \rightarrow K\left[t_{1}, \ldots, t_{m}\right]
$$

induced by

$$
\varphi\left(X_{i}\right)=t_{1}^{a_{1 i}} \cdots t_{m}^{a_{m i}}
$$

The kernel of $\varphi$, denoted by $I_{A}$, is called the toric ideal associated with the matrix $A$.

For toric ideals and toric varieties, see [15] or [14].

Remark 2.1 We can use Macaulay 2 to compute the toric ideal $I_{A}$, cf. [3].

The toric variety determined by the matrix $A$ is the subset of the projective space $\mathbb{P}_{K}^{n}$ given by

$$
X=\left\{\left(t_{1}^{a_{11}} \cdots t_{m}^{a_{m 1}}, \ldots, t_{1}^{a_{1(n+1)}} \cdots t_{m}^{a_{m(n+1)}}\right) \in \mathbb{P}_{K}^{n} \mid t_{1}, \ldots, t_{m} \in K\right\}
$$

Of course, we take the values of $t_{1}, \ldots, t_{m} \in K$ so that they define a point in $\mathbb{P}_{K}^{n}$.

Remark 2.2 This approach generalize many evaluation codes that have been studied before. For instance, if we take the $(n+1) \times(n+1)$ identity matrix, the associated variety becomes the whole projective space and then the corresponding evaluation codes are the Projective Reed-Muller Codes, that are well known (cf. [11], [13]).

On the other hand, if we consider the following $(n+1) \times(n+1)$ matrix

$$
\left(\begin{array}{cccccc}
1 & 1 & 1 & \ldots & 1 & 1 \\
0 & 1 & 0 & \ldots & 0 & 0 \\
0 & 0 & 1 & \ldots & 0 & 0 \\
\ldots & \ldots & \ldots & \ldots & \ldots & \ldots \\
0 & 0 & 0 & \ldots & 1 & 0 \\
0 & 0 & 0 & \ldots & 0 & 1
\end{array}\right)
$$

the corresponding variety becomes the affine space (embedded in the projective space) and the evaluation codes are the Generalized Reed-Muller Codes, which are well known too (cf. [1], [11]). 
In this way, many of the particular cases of the evaluation codes worked before (cf. [2], [4], [5], [6], [7], [12]) can be described with this point of view.

In particular, the evaluation codes arising from Segre's variety, the evaluation codes over the Veronese variety and the Generalized Reed-Solomon codes can be studied with this kind of concepts.

In the following sections we will work with some matrices which have only 1's in the first row and their elements in the other rows are in arithmetic progression.

The 1's in the first row implies that toric ideal is homogeneous in the usual grading.

\section{Main Results}

From now on, we will work with matrices $m \times(n+1)$ of the following form:

$$
\left(\begin{array}{ccccc}
1 & 1 & 1 & \ldots & 1 \\
a_{2} & a_{2}+c_{2} & a_{2}+2 c_{2} & \ldots & a_{2}+n c_{2} \\
a_{3} & a_{3}+c_{3} & a_{3}+2 c_{3} & \ldots & a_{3}+n c_{3} \\
\ldots & \ldots & \ldots & \ldots & \ldots \\
a_{m} & a_{m}+c_{m} & a_{m}+2 c_{m} & \ldots & a_{m}+n c_{m}
\end{array}\right)
$$

Where $a_{2}, \ldots, a_{m}, c_{2}, \ldots, c_{m}$ are positive integers.

With these conditions, the set $X$, which is a projective toric variety, becomes

$$
X=\left\{\left(1, t_{2}^{c_{2}} \cdots t_{m}^{c_{m}}, t_{2}^{2 c_{2}} \cdots t_{m}^{2 c_{m}}, \ldots, t_{2}^{n c_{2}} \cdots t_{m}^{n c_{m}}\right) \mid t_{2}, \ldots, t_{m} \in K^{*}\right\}
$$

If $X=\left\{P_{1}, \ldots, P_{s}\right\} \subset \mathbb{P}_{K}^{n}$, we define, like in the first section of this paper, the evaluation map

$$
\begin{gathered}
e v_{d}: K\left[X_{0}, \ldots, X_{n}\right]_{d} \rightarrow K^{s}, \\
e v_{d}(f)=\left(f\left(P_{1}\right), \ldots, f\left(P_{s}\right)\right)
\end{gathered}
$$

And the linear code $C_{X}(d)$,which is the image of the last evaluation map, will be called the evaluation code of order $d$ defined over the toric variety $X$, or, associated to the matrix $A$.

In the following section we will describe the first parameter of this kind of codes. 


\subsection{The length of the code $C_{X}(d)$}

With the notation used before, the length of the evaluation code defined over the toric variety $X$ is computed in the following theorem.

Theorem 3.1 The length of the evaluation code $C_{X}(d)$, s, is given by

$$
s=\frac{q-1}{\operatorname{gcd}\left(q-1, c_{2}, c_{3}, \ldots, c_{m}\right)}
$$

Proof. Let $K^{*}=\langle a\rangle$ and $t_{i}=a^{j_{i}}$ for all $i=1, \ldots, m$. If we take $c:=\operatorname{gcd}\left(q-1, c_{2}, \ldots, c_{m}\right)$ then

$$
t_{2}^{c_{2}} \cdots t_{m}^{c_{m}}=a^{j_{2} c_{2}+\cdots+j_{m} c_{m}}=\left(a^{c}\right)^{l}
$$

where $j_{2} c_{2}+\cdots+j_{m} c_{m}=c l$.

Moreover, the order of the element $a^{c}$ is given by

$$
\circ\left(a^{c}\right)=\frac{q-1}{c}
$$

And if we take $\alpha=a^{c}$ then

$$
X=\left\{\left(1, \alpha^{l}, \alpha^{2 l}, \ldots, \alpha^{n l}\right): l=1, \ldots, \frac{q-1}{c}\right\}
$$

Therefore $\# X=\frac{q-1}{c}$.

\subsection{The dimension of the code $C_{X}(d)$ and the $a$-invariant of the ideal $I_{X}$}

In this section we will find the dimension of the evaluation codes associated to the matrices defined before and the $a$-invariant of the corresponding vanishing ideal $I_{X}$. We will use the notation that appeared in the proof of Theorem 1 , i.e.,

$$
X=\left\{\left(1, \alpha^{l}, \alpha^{2 l}, \ldots, \alpha^{n l}\right): l=1, \ldots, \frac{q-1}{c}\right\}
$$

where $\alpha=a^{c}, K^{*}=\langle a\rangle, c=\operatorname{gcd}\left(q-1, c_{2}, \ldots, c_{m}\right)$ and $s=\# X=\frac{q-1}{c}$.

On the other hand, we will work with the set $Y$ given by

$$
Y=\left\{\left(1, \alpha^{l}\right) \in \mathbb{P}_{K}^{1}: l=1, \ldots, s\right\}
$$

Then $s=\# X=\# Y$. Also, we will use the following notation: $S=$ $K\left[X_{0}, \ldots, X_{n}\right], S_{1}=K\left[Y_{0}, Y_{1}\right]$, and $I_{X}=\langle f \in S: f(P)=0$ for all $P \in X\rangle$ and 


$$
I_{Y}=\left\langle g \in S_{1}: g(Q)=0 \text { for all } Q \in Y\right\rangle .
$$

The corresponding homogeneous vanishing ideals.

Lemma 3.2 The vanishing ideal $I_{Y}$ is given by

$$
I_{Y}=\left\langle Y_{1}^{s}-Y_{0}^{s}\right\rangle
$$

Proof. Let $F$ be an homogeneous polynomial in the ideal $I_{Y}$. If we take the lexicographic order $Y_{1}>Y_{0}$ and we apply the division algorithm we obtain that

$$
F=G \cdot\left(Y_{1}^{s}-Y_{0}^{s}\right)+r
$$

where $G, r \in S_{1}$ and $r=0$ or $r$ is a $K$-linear combination of monomials none of which is divisible by $Y_{1}^{s}$. Therefore, $\operatorname{deg}_{Y_{1}} r<s$. But $0=F\left(1, \alpha^{l}\right)=r\left(1, \alpha^{l}\right)$ for all $l=1, \ldots, s$ and it means that $r$ has at least $s$ roots (viewed as a polynomial in the variable $Y_{1}$ ). This contradicts the fact that $\operatorname{deg}_{Y_{1}} r<s$.

Lemma 3.3 The a-invariant of the ideal $I_{Y}$ is given by

$$
a_{Y}=s-2
$$

Proof. From Lemma 1 we conclude that $s$ is the lowest degree of a nontrivial homogeneous component of the ideal $I_{Y}$ and then $H_{Y}(d)=\left(\begin{array}{c}d+1 \\ d\end{array}\right)=$ $d+1$ if $d<s$. Therefore $H_{Y}(s-2)=s-1<\# Y$ and $H_{Y}(s-1)=s=\# Y$. It proves that $a_{Y}=s-2$.

Theorem 3.4 The dimension of the evaluation code $C_{X}(d)$ is given by

$$
\operatorname{dim}_{K} C_{X}(d)=n d+1 \text { if } n d<s
$$

Proof. We can define the following map

$$
\begin{aligned}
\psi: S_{d} & \longrightarrow S_{1}(n d) \\
f\left(X_{0}, \ldots, X_{n}\right) & \longrightarrow f\left(Y_{0}^{n}, Y_{0}^{n-1} Y_{1}, \ldots, Y_{1}^{n}\right)
\end{aligned}
$$

We will show that this map is a surjective map. It is enough if we work with monomials of the form $Y_{0}^{n d-i} Y_{1}^{i}$ for $i=0, \ldots, n d$. If $i \leq d$ it is easy to see that $\psi\left(X_{0}^{d-i} X_{1}^{i}\right)=Y_{0}^{n d-i} Y_{1}^{i}$ and if $i>d$ with $i=d q_{1}+r_{1}$, with $0 \leq r_{1}<d$ then $\psi\left(X_{q_{1}}^{d-r_{1}} X_{q_{1}+1}^{r_{1}}\right)=Y_{0}^{n d-i} Y_{1}^{i}$. Therefore, $\psi$ is a surjective map.

It is easy to see that $\operatorname{Ker} \psi=I_{X}(d)$ and then

$$
S_{d} / I_{X}(d) \cong S_{1}(n d)
$$

Therefore $H_{X}(d)=H_{Y}(n d)$. Finally, $H_{X}(d)=n d+1$ if $n d<s$ and in consequence $\operatorname{dim}_{K} C_{X}(d)=n d+1$ if $n d<s$. 
Remark 3.5 In the proof of the last theorem we showed that if $n d<s$ then $S_{d} / I_{X}(d) \cong S_{1}(n d)$, but due to the fact that $I_{Y}(n d)=\{0\}$ (because by Lemma $1 \mathrm{~s}$ is the lowest degree of a nontrivial homogeneous component of the ideal $I_{Y}$ ) we obtain that $S_{d} / I_{X}(d) \cong S_{1}(n d) / I_{Y}(n d)$ and it proves that $H_{X}(d)=H_{Y}(n d)$ if $n d<s$. Moreover, the induced isomorphism between $C_{X}(d)$ and $C_{Y}(n d)$ preserves the weight of the codewords, i.e., the minimum distance of $C_{X}(d)$ is equal to the minimum distance of $C_{Y}(n d)$.

Corollary 3.6 The a-invariant of the ideal $I_{X}$ is given by

$$
a_{X}= \begin{cases}j-1 & \text { if } r=0 \\ j & \text { if } 0<r<n\end{cases}
$$

where $j$ is the quotient and $r$ is the remainder when we divide $s-1$ over $n$, i.e., $s-1=n j+r$ with $0 \leq r<n$.

Proof. If $0<r<n$ then $H_{X}(j+1)=H_{Y}(n j+n)=H_{Y}(s+n-r-1)$. But $n-r-1 \geq 0$ and then $H_{X}(j+1)=s$. Moreover, $H_{X}(j)=H_{Y}(n j)=$ $H_{Y}(s-r-1)$. But, by Lemma $2, s-r-1 \leq s-2=a_{Y}$ and then $H_{X}(j)<s$. It proves that $a_{X}=j$ if $0<r<n$.

If $r=0, H_{X}(j)=H_{Y}(n j)=H_{Y}(s-1)=s$. On the other hand, $H_{X}(j-1)=$ $H_{Y}(n j-n)=H_{Y}(s-n-1)<s$. Therefore $a_{X}=j-1$ if $r=0$.

\subsection{The minimum distance of the code $C_{X}(d)$}

In this section we will find the minimum distance of the code $C_{X}(d)$ and we will note that this code is an MDS code.

Theorem 3.7 If $n d<s$ then the minimum distance of the code $C_{X}(d), \delta$, is given by

$$
\delta=s-n d
$$

Proof. We know that, by the Singleton bound, $(n d+1)+\delta \leq s+1$ and then $\delta \leq s-n d$.

On the other hand, by Remark $3, \delta$ is the minimum distance of the code $C_{Y}(n d)$. It is enough if we show that any codeword in the code $C_{Y}(n d)$ has Hamming weight at least $s-n d$. Let $G\left(Y_{0}, Y_{1}\right) \in S_{1}(n d)$. Then $G\left(1, X_{1}\right)$ has degree $g \leq n d$ and therefore it has $g$ or less roots. If $\Lambda$ is the codeword associated to the polynomial $G\left(Y_{0}, Y_{1}\right)$, i.e,

$$
\Lambda=\left(G(1, \alpha), G\left(1, \alpha^{2}\right), \ldots, G\left(1, \alpha^{s}\right)\right)
$$

then

$$
w(\Lambda) \geq s-g \geq s-n d
$$

Where $w(\Lambda)$ is the Hamming weight of the codeword $\Lambda$. Then $\delta \geq s-n d$. 


\subsection{Two examples}

In this section we will give two examples of the evaluation codes defined in the previous sections and with the help of Macaulay 2 (cf. [8]), we will compute the main parameters of these codes (length, dimension and minimum distance) and other characteristics of the toric variety $X$ ( $a$-invariant, Hilbert series, vanishing ideal).

\subsubsection{Example I}

In this example we will work with a field with 81 elements and with a $3 \times 6$ matrix in order to illustrate the main results.

Let $K=G F\left(3^{4}\right)=\left\{0,1, a, a^{2}, \ldots, a^{79}\right\}$ be the field with 81 elements and consider the following matrix

$$
A=\left(\begin{array}{cccccc}
1 & 1 & 1 & 1 & 1 & 1 \\
2 & 10 & 18 & 26 & 34 & 42 \\
5 & 33 & 61 & 89 & 117 & 145
\end{array}\right)
$$

In this case, $q=81, c_{2}=8, c_{3}=28$ and then

$$
s=\# X=\frac{80}{\operatorname{gcd}(80,8,28)}=20
$$

Furthermore, the projective toric variety $X \subset \mathbb{P}_{K}^{5}$ is:

$$
\begin{aligned}
& X=\left\{(1,1,1,1,1,1),\left(1, a^{28}, a^{56}, a^{4}, a^{32}, a^{60}\right),\right. \\
& \left(1, a^{56}, a^{32}, a^{8}, a^{64}, a^{40}\right),\left(1, a^{4}, a^{8}, a^{12}, a^{16}, a^{20}\right), \\
& \left(1, a^{32}, a^{64}, a^{16}, a^{48}, 1\right),\left(1, a^{60}, a^{40}, a^{20}, 1, a^{60}\right), \\
& \left(1, a^{8}, a^{16}, a^{24}, a^{32}, a^{40}\right),\left(1, a^{36}, a^{72}, a^{28}, a^{64}, a^{20}\right), \\
& \left(1, a^{64}, a^{48}, a^{32}, a^{16}, 1\right),\left(1, a^{12}, a^{24}, a^{36}, a^{48}, a^{60}\right), \\
& \left(1, a^{40}, 1, a^{40}, 1, a^{40}\right),\left(1, a^{68}, a^{56}, a^{44}, a^{32}, a^{20}\right), \\
& \left(1, a^{16}, a^{32}, a^{48}, a^{64}, 1\right),\left(1, a^{44}, a^{8}, a^{52}, a^{16}, a^{60}\right), \\
& \left(1, a^{72}, a^{64}, a^{56}, a^{48}, a^{40}\right),\left(1, a^{20}, a^{40}, a^{60}, 1, a^{20}\right), \\
& \left(1, a^{48}, a^{16}, a^{64}, a^{32}, 1\right),\left(1, a^{76}, a^{72}, a^{68}, a^{64}, a^{60}\right), \\
& \left.\left(1, a^{24}, a^{48}, a^{72}, a^{16}, a^{40}\right),\left(1, a^{52}, a^{24}, a^{76}, a^{48}, a^{20}\right)\right\}
\end{aligned}
$$

Moreover, $n=5, s-1=19=5(3)+4$ and then $j=3$ and $r=4$. Therefore the $a$-invariant is $j=3$ and we can work with the codes $C_{X}(2)$ and $C_{X}(3)$ with dimensions $H_{X}(2)=5(2)+1=11$ and $H_{X}(3)=5(3)+1=16$, respectively.

By Theorem 3 the minimum distance of the code $C_{X}(2)$ is $\delta=20-5(2)=10$ and the corresponding value for the code $C_{X}(3)$ is $\delta=20-5(3)=5$.

In fact, the Hilbert Series is given by 


$$
F_{X}(t)=\frac{1+4 t-t^{4}-4 t^{5}}{(1-t)^{2}}
$$

Furthermore, if we apply the algorithm used in [3], pp. 179-182, we obtain that

$$
\begin{gathered}
I_{\bar{A}}=\left\langle X_{4}^{2}-X_{3} X_{5}, X_{3} X_{4}-X_{2} X_{5}, X_{2} X_{4}-X_{1} X_{5}, X_{1} X_{4}-X_{0} X_{5}, X_{3}^{2}-\right. \\
\left.X_{1} X_{5}, X_{2} X_{3}-X_{0} X_{5}, X_{1} X_{3}-X_{0} X_{4}, X_{2}^{2}-X_{0} X_{4}, X_{1} X_{2}-X_{0} X_{3}, X_{1}^{2}-X_{0} X_{2}\right\rangle
\end{gathered}
$$

Where $I_{\bar{A}}$ means the toric ideal over the matrix $A$ if we work with the algebraic closure, $\bar{K}$, of the field $K$.

Finally, the vanishing ideal of the coordinate ring of the toric variety $X$ is given by

$$
I_{X}=\left\langle I_{\bar{A}},\left\{X_{0}^{4}-X_{5}^{4}\right\}\right\rangle
$$

\subsubsection{Example II}

In this example we will work with a field with 32 elements and with a $3 \times 4$ matrix.

Let $K=G F\left(2^{5}\right)=\left\{0,1, a, a^{2}, \ldots, a^{30}\right\}$ be the field with 32 elements and the matrix $A$ given by

$$
A=\left(\begin{array}{cccc}
1 & 1 & 1 & 1 \\
7 & 13 & 19 & 25 \\
4 & 14 & 24 & 34
\end{array}\right)
$$

In this case $q=32, c_{2}=6, c_{3}=10$ and then

$$
s=\# X=\frac{31}{\operatorname{gcd}(31,6,10)}=31
$$

Moreover, the toric variety $X \subset \mathbb{P}_{K}^{3}$ is

$$
\begin{aligned}
& X=\left\{(1,1,1,1),\left(1, a^{10}, a^{20}, a^{30}\right),\left(1, a^{20}, a^{9}, a^{29}\right),\right. \\
& \left(1, a^{29}, a^{27}, a^{25}\right),\left(1, a^{8}, a^{16}, a^{24}\right),\left(1, a^{18}, a^{5}, a^{23}\right), \\
& \left(1, a^{28}, a^{25}, a^{22}\right),\left(1, a^{7}, a^{14}, a^{21}\right),\left(1, a^{17}, a^{3}, a^{20}\right), \\
& \left(1, a^{27}, a^{23}, a^{19}\right),\left(1, a^{6}, a^{12}, a^{18}\right),\left(1, a^{16}, a, a^{17}\right), \\
& \left(1, a^{23}, a^{15}, a^{7}\right),\left(1, a^{2}, a^{4}, a^{6}\right),\left(1, a^{12}, a^{24}, a^{5}\right), \\
& \left(1, a^{22}, a^{13}, a^{4}\right),\left(1, a, a^{2}, a^{3}\right),\left(1, a^{11}, a^{22}, a^{2}\right) \\
& \left(1, a^{24}, a^{17}, a^{10}\right),\left(1, a^{3}, a^{6}, a^{9}\right),\left(1, a^{13}, a^{26}, a^{8}\right), \\
& \left(1, a^{30}, a^{29}, a^{28}\right),\left(1, a^{9}, a^{18}, a^{27}\right),\left(1, a^{19}, a^{7}, a^{26}\right), \\
& \left(1, a^{25}, a^{19}, a^{13}\right),\left(1, a^{4}, a^{8}, a^{12}\right),\left(1, a^{14}, a^{28}, a^{11}\right), \\
& \left(1, a^{26}, a^{21}, a^{16}\right),\left(1, a^{5}, a^{10}, a^{15}\right),\left(1, a^{15}, a^{30}, a^{14}\right)
\end{aligned}
$$




$$
\left.\left(1, a^{21}, a^{11}, a\right)\right\}
$$

On the other hand, $n=3, s-1=30=3(10)$ and then $j=10$ and $r=0$. Therefore the $a$-invariant is $j-1=9$ and we can work with the codes $C_{X}(i)$ with dimensions $H_{X}(i)=3 i+1$ for $i=2, \ldots, 9$.

By Theorem 3 we obtain that the minimum distance of the codes $C_{X}(i)$ is given by $\delta_{i}=31-3 i$ for $i=2, \ldots, 9$ and all of them are MDS codes.

In fact, the Hilbert series is

$$
F_{X}(t)=\frac{1+2 t-3 t^{11}}{(1-t)^{2}}
$$

In the same way that in the last example we obtain

$$
I_{\bar{A}}=\left\{X_{2}^{2}-X_{1} X_{3}, X_{1} X_{2}-X_{0} X_{3}, X_{1}^{2}-X_{0} X_{2}\right\}
$$

Furthermore, the vanishing ideal of the coordinate ring of the toric variety $X$ is given by

$$
I_{X}=\left\langle I_{\bar{A}},\left\{X_{0}^{11}-X_{1} X_{3}^{10}, X_{0}^{10} X_{1}-X_{2} X_{3}^{10}, X_{0}^{10} X_{2}-X_{3}^{11}\right\}\right\rangle
$$

\section{References}

[1] P. Delsarte, J.M. Goethals, F.J. MacWilliams. On generalized ReedMuller codes and their relatives, Inform. and Control, vol. 16, pp. 403-422 (1970).

[2] I. Duursma, C. Rentería and H. Tapia-Recillas. Reed Muller codes on Complete Intersections. Applicable Algebra in Engineering, Communication and Computing, AAECC, Springer, vol. 11, pp. 455-462 (2001).

[3] D. Eisenbud, D.R Grayson, M. Stillman, B. Sturmfels. Computations in Algebraic Geometry with Macaulay 2. Springer Verlag (2002).

[4] M. González-Sarabia, C. Rentería and H. Tapia-Recillas. Reed-MullerType Codes Over the Segre Variety. Finite Fields and their Applications, vol. 8, pp. 511-518 (2002).

[5] M. González-Sarabia, C. Rentería and M.A. Hernández de la Torre. Minimum distance and second generalized Hamming weight of two particular linear codes. Congressus Numerantium, 161, pp. 105-116 (2003).

[6] M. González-Sarabia, C. Rentería. The dual code of some Reed-Mullertype codes. Applicable Algebra in Engineering, Communication and Computing, AAECC, Springer, 14, pp. 329-333 (2004). 
[7] M. González-Sarabia, C. Rentería. The Dual Code Arising From Segre's Variety. Congressus Numerantium, 174, pp. 199-205 (2005).

[8] D.R. Grayson, M. Stillman: Macaulay 2 (1998).

[9] G. Lachaud, The parameters of the Projective Reed-Muller codes, Discrete Mathematics, vol 81, pp. 217-221 (1990).

[10] C. Rentería, H. Tapia-Recillas, Linear codes associated to the ideal of points in $\mathbb{P}^{d}$ and its canonical module, Communications in Algebra 24 (3), pp. 1083-1090 (1996).

[11] C. Rentería, H. Tapia-Recillas, Reed-Muller codes: An ideal Theory Approach, Communications in Algebra, 25 (2), pp. 401-413 (1997).

[12] C. Rentería, H. Tapia-Recillas, The $a$ - invariant of some Reed-Muller Codes, Applicable Algebra in Engineering, Communication and Computing, AAECC, Springer vol. 10, No. 1, pp. 33-40 (1999).

[13] A.B. Sörensen, Projective Reed-Muller Codes, IEEE Transactions on Information Theory, vol. 37, No. 6, pp. 1567-1576 Nov. (1991).

[14] B. Sturmfels, Gröbner bases and convex polytopes. American Mathematical Society, Providence, RI, 1996.

[15] R.H. Villarreal, Monomial Algebras, Monographs and Textbooks in pure and Applied Mathematics, Marcel Dekker, inc, New York, 2001.

Received: November 3, 2006 